\title{
ZBED1/DREF: A transcription factor that regulates cell proliferation (Review)
}

\author{
YARONG JIN ${ }^{1,2}$, RUILEI LI ${ }^{2}$, ZHIWEI ZHANG ${ }^{2,3}$, JINJIN REN $^{1}$, XIN SONG $^{2}$ and GONG ZHANG ${ }^{1}$ \\ ${ }^{1}$ Department of Radiotherapy, People's Hospital of Shanxi Province, Taiyuan, Shanxi 030012; ${ }^{2}$ Department of Cancer \\ Biotherapy Center, The Third Affiliated Hospital of Kunming Medical University (Tumor Hospital of Yunnan Province), \\ Kunming, Yunnan 650118; ${ }^{3}$ Department of Oncology, Affiliated Hospital of \\ Hebei University of Engineering, Handan, Hebei 056002, P.R. China
}

Received March 7, 2020; Accepted July 7, 2020

DOI: $10.3892 / \mathrm{ol} .2020 .11997$

\begin{abstract}
Maintenance of genomic diversity is critically dependent on gene regulation at the transcriptional level. This occurs via the interaction of regulatory DNA sequence motifs with DNA-binding transcription factors. The zinc finger, BED-type (ZBED) gene family contains major DNA-binding motifs present in human transcriptional factors. It encodes proteins that present markedly diverse regulatory functions. ZBED1 has similar structural and functional properties to its Drosophila homolog DNA replication-related element-binding factor (DREF) and plays a critical role in the regulation of transcription. ZBED1 regulates the expression of several genes associated with cell proliferation, including cell cycle regulation, chromatin remodeling and protein metabolism, and some genes associated with apoptosis and differentiation. In the present review, the origin, structure and functional role of ZBED1 were comprehensively assessed. In addition, the similarities and differences between ZBED1 and its Drosophila homolog DREF were highlighted, and future research directions, particularly in the area of clinical cancer, were discussed.
\end{abstract}

\section{Contents}

1. Introduction

2. Origin of ZBED1

Correspondence to: Dr Gong Zhang, Department of Radiotherapy, People's Hospital of Shanxi Province, 29 Shuangta, Taiyuan, Shanxi 030012, P.R. China

E-mail: zhanggong7446@163.com

Dr Xin Song, Department of Cancer Biotherapy Center, The Third Affiliated Hospital of Kunming Medical University (Tumor Hospital of Yunnan Province), 519 Kunzhou Road, Xishan, Kunming, Yunnan 650118, P.R. China

E-mail: songxin_68@163.com

Key words: transcription factor, ZBED1, DREF, proliferation, transposable element
3. Similar zinc-finger structure of DREF and ZBED1

4. Multiple functions of DREF and ZBED1

5. ZBED1 as a transcription factor for cell proliferation

6. Conclusions and future perspectives

\section{Introduction}

Multicellular organisms develop complex genomes through transcriptional regulation, which is crucial for spatial and temporal cellular specialization to promote phenotypic complexity. The interaction of regulatory DNA sequence motifs with DNA-binding transcription factors induces transcriptional regulation. These transcriptional factors serve a critical role in the activation or repression of target genes. Zinc-finger proteins are among the most commonly occurring DNA-binding motifs and constitute 2-3\% of the transcription factors in the human genome (1). These proteins are associated with several biological functions, including differentiation, chromatin remodeling and development (2). The zinc finger, BED-type (ZBED) gene family is widely expressed in vertebrate tissues. It comprises a closely related group of genes that contribute to the regulation of various functions by encoding regulatory proteins. For example, the ZBED6 gene is involved in the regulation of diverse phenotypic effects. ZBED6 binds to the conserved target motif of insulin-like growth factor 2 and inhibits its expression, thereby promoting cell proliferation, growth and development in placental mammals (3). Furthermore, conflicting phenotypic changes were induced by the inactivation of ZBED6 in HCT116 and RKO human colorectal cancer cell lines, with consequences of reduced and increased growth, respectively, indicating that ZBED6 exhibits transcriptional modulating properties, and that the effect of ZBED6 on tumor development is dependent on the transcriptional state and genetic background of its target genes (4). The ZBED3 protein interacts with axin, which is vital for $\mathrm{Wnt} / \beta$-catenin signal modulation in mammalian carcinogenesis and embryogenesis (5). Notably, Fan et al (6) reported higher expression levels of ZBED3 in cancer tissues compared with normal lung tissues and demonstrated that ZBED3 expression levels have clinicopathological significance. Downregulation 
of ZBED3 by small interfering RNA significantly inhibits expression of p120ctn-1 and $\beta$-catenin in lung cancer cells; these results indicate that lung cancer cell invasion may be induced by ZBED3, which functions by regulating p120ctn-1 and $\beta$-catenin (6). Therefore, ZBED3 has potential application in the management of cancer, particularly non-small cell lung cancer. Furthermore, ZBED4 is restricted in glial Müller cells and the cone photoreceptors of the human retina (7). It binds to DNA and RNA sequences and effectively affects the transcription process of genes expressed in the retina via G-rich promoters (8).

ZBED1 was identified as a human homolog of Drosophila DNA replication-related element-binding factor (DREF) via BLAST search. Also known as human DREF, Activator (Ac)-like transposable element, TRAMP and KIAA0785, ZBED1 has been found to play a critical role in cell proliferation via the regulation of gene expression (9). As ZBED1 has similar structural and functional properties to DREF, the present review comprehensively describes recent progress on the origin, structure and functions on ZBED1, specifically highlights the similarities and differences between ZBED1 and DREF, and discusses future research directions.

\section{Origin of ZBED1}

A large proportion of vertebrate genomes consist of eukaryotic genome components and transposable elements (TEs), such as DNA transposons and retrotransposons (10-12). Several genes, particularly those that possess rapidly evolving coding sequence properties, have been shown to contain functionally important TEs that are involved in gene expression and regulatory changes $(11,13,14)$. Notably, a quarter of analyzed human promoter regions have been found to have TE-derived sequences that potentially function as alternative promoters in several genes (13). TEs contribute entire functional genes to the host genome through molecular domestication $(11,15,16)$. Domesticated TEs, which are immobile, often exist as single-copy orthologs within the genomes of associated organisms (15). Since DNA transposons encode multi-domain proteins with diverse functions, such as protein- and DNA-binding affinity, they may be suitable for domestication to perform host functions.

The hAT transposons and their associated domesticated sequences form a large superfamily that is divided into Buster and Ac families. These two families exhibit distinct target-site selections and were named according to the first identified transposon or transposon-like sequence in each family (17). Through phylogenetic methodology, Hayward et al (18) demonstrated that ZBED5, ZBED7, ZBED8 and ZBED9 are associated with the Buster family and are distinct from other ZBEDs. The remaining ZBEDs form two monophyletic clades within the Ac transposon family. Notably, ZBED2, ZBED3, ZBED4, ZBED6 and ZBEDX form a monophyletic group with C7ORF29 and differ from ZBED1 (Fig. 1). The ZBEDs exhibit structural similarity via the zinc finger domain that functions in DNA binding. Closely related ZBED molecules regulate a variety of different host functions, indicating that ZBED protein domains are particularly suitable for controlling host functions.

\section{Similar zinc-finger structure of DREF and ZBED1}

A palindromic 8 base-pair (bp) sequence 5'-TATCGATA was identified as being common to the promoter regions of the proliferating cell nuclear antigen (PCNA) and DNA polymerase $\alpha$ genes in Drosophila (19). This sequence is known as the DNA replication-related element (DRE) and is vital for transcriptional activation. Subsequently, several methods, including band mobility shift assays, were used to identify specific DREF (20), and the cDNA of DREF was then cloned (21). Notably, DREF was found to comprise a polypeptide with 709 amino acid (aa) residues. The DREF gene of Drosophila virilis (D. virilis) has been shown to share $71 \%$ of its aa sequence identity with its D. melanogaster homolog, with three highly conserved regions (CRs) at the aa positions 14-182 (CR1; identity, 86.4\%), 432-568 (CR2; identity, 86.1\%) and 636-730 (CR3; identity, 83.3\%) of the D. virilis DREF (22).

DREF is proposed to have originated from a combination of the amino-terminal boundary element-associated factor (BEAF) and DREF (BED) zinc finger domain protein and the carboxy-terminal hATC domain (23). Aravind (24) conducted sequence analyses that led to the prediction of protein signature $\mathrm{C}_{\mathrm{X} 2} \mathrm{C}_{\mathrm{XN}} \mathrm{H}_{\mathrm{X} 3-5}(\mathrm{H} / \mathrm{C})$ for the BED finger, a name derived from DREF protein and domesticated Drosophila BEAF. The BED domain forms the zinc finger common to fungal, animal and plant proteins. This domain has been postulated to originate from transposons in cellular genes or to have been acquired for the cellular functions of transposases at independent occasions. A domain of BED zinc finger containing 50-60 aa residues has been found to contain a characteristic motif with two highly conserved aromatic residues. It has a shared pattern of histidines and cysteines that is considered to create a DNA-binding zinc finger domain. Furthermore, the BED domains of BEAF and DREF have been reported to exhibit DNA-binding properties $(21,25)$.

ZBED1 comprises a polypeptide of 694 aa residues that has $21 \%$ similarity and $22 \%$ identity when compared with DREF (9). Three CRs between D. virilis and D. melanogaster as aforementioned have aa sequences highly similar to those of the ZBED1 polypeptide, particularly the CR1 region, which is essential for DRE-binding and dimerization. The ZBED1 binding sequence [hDRE; 5'-TGTCG(C/T)GA(C/T)A] was identified using the CASTing method and found to overlap with the DREF (5'-TATCGATA) sequence (9). Furthermore, the 6-bp sequence 5'-TCG(C/T)GA at the center of ZBED1 was indicated to serve an important role in ZBED1 binding (9). Two regions of ZBED1, CR1 and CR3, correspond with two domains that are conserved within the family, namely the amino-terminal BED zinc finger (aa 23-72) and the carboxyl-terminal hATC domain (26). The BED zinc finger is regarded as a DNA-binding domain of chromatin boundary element-binding transposase (24), whereas the hATC domain of hAT transposase family members is considered as the dimerization domain, as assessed through in vitro cross-linking experiments and yeast two-hybrid assays using Ac and housefly Hermes family members $(27,28)$.

The DNA-binding protein ZBED1 has granular structures and is predominantly distributed in the nucleus (9). A study by Yamashita et al (26) explored the mechanism of action of ZBED1 using detailed mutagenesis analyses to investigate 


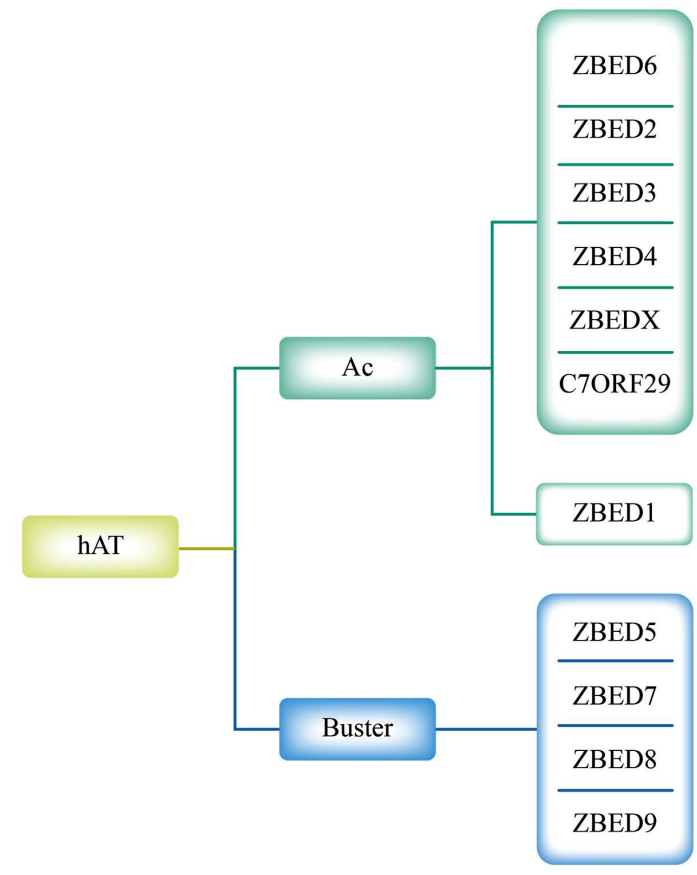

Figure 1. Phylogenetic relationships of ZBED genes with related sequences. The hAT transposons comprise Buster and Ac families. ZBED6, ZBED2, ZBED3, ZBED4, ZBEDX, C7ORF29, and ZBED1 belong to the Ac family. ZBED5, ZBED7, ZBED8, and ZBED9 belong to the Buster family. ZBED, zinc finger, BED-type; Ac, Activator.

the functions of the hATC domain. Trp-590, Trp-591 and Leu-601, which are highly conserved hydrophobic aa residues in hATC domains, were found to be crucial for the in vivo self-association of ZBED1. Furthermore, through substitution and truncated mutant evaluation, it was found that the hATC domain (aa 571-651) has two regions with distinct functions. The first region, namely the amino-terminal region aa 571-624, is required for various processes, including ZBED1 DNA-binding, nuclear accumulation and self-association. The second region, namely the carboxyl-terminal region aa 625-651, is vital for granular pattern formation and self-association. These findings indicate that ZBED1 self-association via the hATC domain is required for nuclear accumulation prior to nuclear importation. Furthermore, the aa 652-694 region has been identified to contain highly ordered complexes of ZBED1 binding sequences, whereas the aa 520-551 region is a classical nuclear localization signal (Fig. 2).

\section{Multiple functions of DREF and ZBED1}

Matsukage et al (29) used bioinformatics analysis to determine the type and number of genes regulated by the DRE/DREF system. They established that 456 DRE sequences and 156 genes with DRE sequences are $<1 \mathrm{~kb}$ upstream of their transcription initiation sites. Most of these genes are associated with processes required for cell proliferation (30), including chromatin remodeling, cell cycle regulation and protein metabolism, while others are associated with apoptosis and differentiation. This indicates that the DRE/DREF system functions as a regulator of several cell proliferation-associated genes (Fig. 3). Ohshima et al (9) searched the GenBank $^{\circledR}$ and EMBL databases to identify genes controlled by ZBED1 protein. Through BLAST analysis, they found $>500 \mathrm{TGTCG}(\mathrm{C} / \mathrm{T}) \mathrm{GA}(\mathrm{T} / \mathrm{C}) \mathrm{A}-$ like sequences in the human genome. Notably, ZBED1 binding sequences exist in a series of genes involved in DNA synthesis (DNA replication) and repair, protein synthesis, regulation of chromatin structure and cell cycle regulation, which seems to be consistent with DREF (31), as some of these genes have been shown to be controlled by the DRE/DREF system (20,32-35). These findings indicate that ZBED1 is a potential functional homolog of Drosophila DREF and can regulate the expression of genes involved in cell proliferation via a similar mechanism.

A notable study indicates that DREF preferentially binds to and activates housekeeping enhancers that are located closely to ubiquitously expressed genes with specificity to its core promoter, and suggests that the DRE motif is required and sufficient for housekeeping enhancer function (36). The CGCG sequence is central to the ZBED1 binding sequence TGTCGCGACA that occurs mostly in promoter regions of housekeeping genes within the mammalian genome, suggesting that ZBED1 could regulate numerous housekeeping genes with $\mathrm{CpG}$ islands (37).

Chromatin remodeling. Chromatin-remodeling protein contains many conserved motifs and is encoded by human alpha-thalassemia and mental retardation X-linked syndrome (hATRX) gene. The ATRX protein contains a helicase/ATPase domain at its carboxyl terminus, which has catalytic subunits of chromatin remodeling complexes (38). Valadez-Graham et al (39) found that dATRX, a Drosophila homolog of hATRX, interacts with transcription factor DREF in vivo and in vitro. This results in transcriptional regulation of the pannier (pnr) gene, which belongs to the GATA family of transcription factors. Therefore, the transcriptional factor DREF is proposed to associate with the chromatin remodeling factor dATRX in the regulation of the pnr gene. These studies on Drosophila may provide insights for further research exploring the functional interaction of hATRX with ZBED1 in humans.

Studies conducted by Hart et al $(25,40)$ suggested that DREF functions as a BEAF antagonist that is necessary for boundary activity of the special chromatin structure region of the Drosophila 87A7 hsp70 gene $(25,40,41)$. The boundary element sequence 5'-CGATA-3', which is also present in DRE (5'-TATCGATA-3'), binds to BEAF-32. This induces the blockade of upstream regulatory elements such as enhancers (25), as demonstrated by chromatin immunoprecipitation. Thus, it is suggested that BEAF may bind to the same sequences as DREF and binding sequence competition between BEAF and DREF may be crucial in the regulation of chromatin boundary activities.

Drosophila Mi-2 (dMi-2), a nucleosome-stimulated ATPase, employs energy from ATP hydrolysis to deacetylate nucleosomal histones and mobilize histone octamers in certain cases (42). A study revealed that dMi-2 negatively regulates DREF by inhibiting DNA binding activity when the C-terminal region of dMi-2 binds to DREF (43). The chromatin remodeling factor $\mathrm{Mi}-2$ has also been reported to be a ZBED1-interacting protein (43). The above findings indicate that the activity of DREF is regulated via protein complexes that determine the chromatin structure. 
$\mathrm{BED}(\mathrm{CR} 1)$

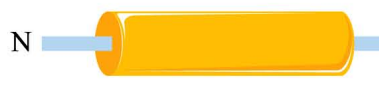

$123 \quad 72$

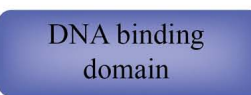

72

520
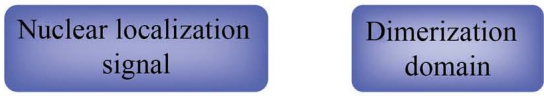

$624 \quad 651$

694

Figure 2. Structural representation of ZBED1 protein. Different colors represent different protein domains. Numbers correspond to the amino acid positions of estimated domain boundaries. ZBED1, zinc finger, BED-type 1; CR, conserved region.

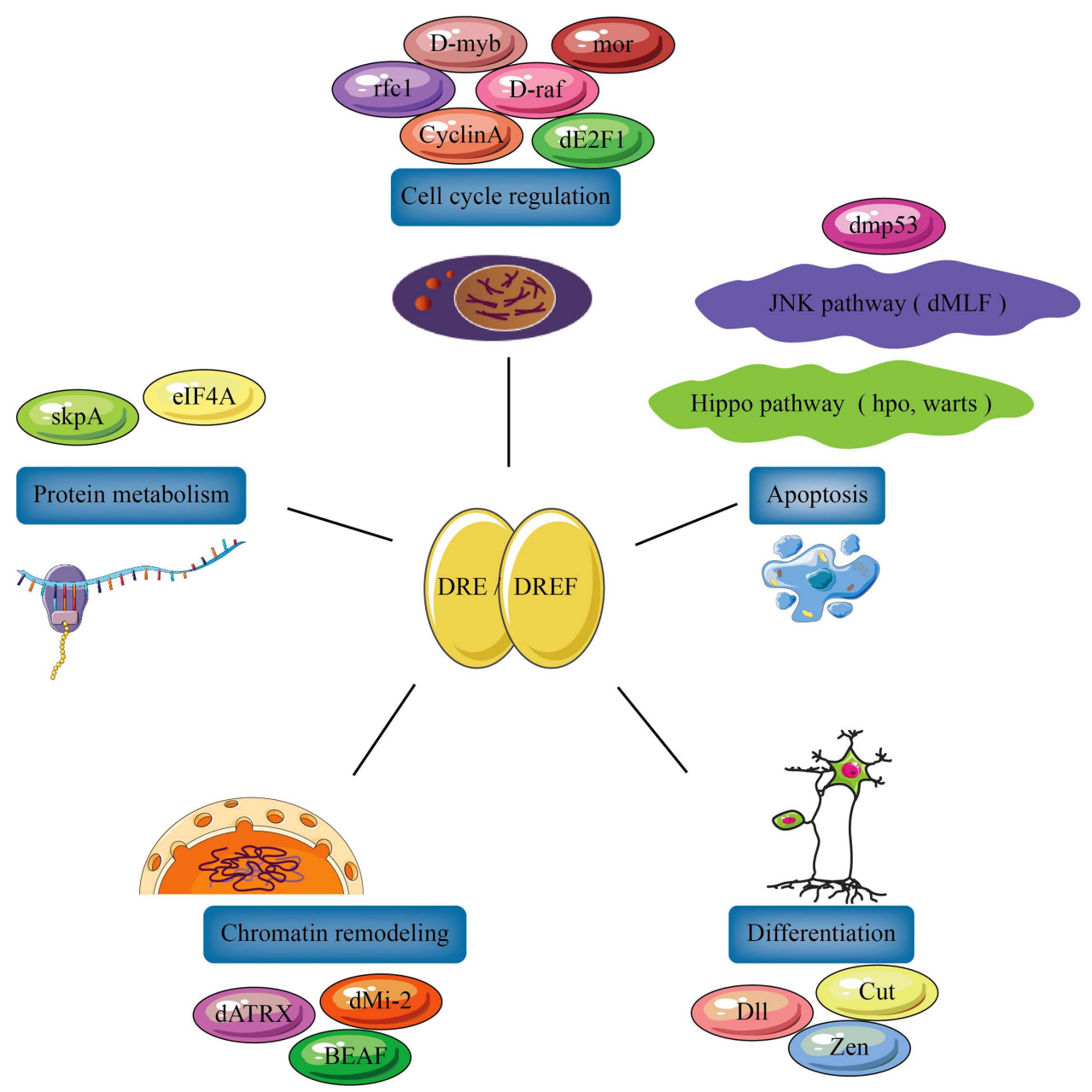

Figure 3. Regulatory network of transcription factor DREF. The genes regulated by DRE/DREF are associated with chromatin remodeling, cell cycle regulation, protein metabolism, apoptosis and differentiation. DRE, DNA replication-related element; DREF, DNA replication-related element-binding factor.

Protein metabolism. DREF has been indicated to directly regulate the expression of the eukaryotic initiation factor $4 \mathrm{~A}$ (eIF4A) gene (44). The eIF4A gene belongs to the DEAD-box family of ATP-dependent RNA helicases (45) and is proposed to function by unwinding the secondary structure of 5'-untranslated regions of mRNA in the cap $\left(\mathrm{m}^{7} \mathrm{GpppN}\right)$-dependent initiation of protein synthesis $(46,47)$. Furthermore, eIF4A plays a significant regulatory role in the initiation of translation (48). A study by Ida et al (44) showed that eIF4A gene promoter activity and eIF4A mRNA levels decreased following the knockdown of DREF in Drosophila S2 cells. Also, through a band mobility shift assay, DRE sequences in the eIF4A gene promoter were found to bind with DREF in vitro. These findings together indicate that the eIF4A gene is regulated via the DREF pathway and that, therefore, DREF regulates protein synthesis, particularly by initiating translation.

DREF has been found to bind to the skpA gene locus via DRE sequences. DRE1 and DRE2 sites present in the 5 '-flanking region of the skpA gene have been reported to serve vital roles in skpA promoter activity in living flies and cultured cells (49). SKP1 protein is a key constituent of the SKP1, cullin/CDC53, F-box protein (SCF) complex and functions in protein degradation, linking the substrate recognition subunit F-box protein to a cullin that then binds the 
ubiquitin-conjugating enzyme. Notably, the SKP1 constituent among Drosophila SCF ubiquitin ligases is referred to as skpA (50). Further study has shown that the knockdown of DREF in tissues with highly distributed skpA abrogates the skpA gene expression whereas the overexpression of DREF activates endogenous skpA expression, indicating that skpA may be effectively regulated via the DRE/DREF pathway at the transcriptional level (49). The available data, therefore, indicate that DREF enhances protein degradation and protein synthesis by activating genes involved in various processes, presumably through active protein metabolism in proliferating cells.

Cell cycle regulation. A study revealed via luciferase transient expression assays that DREs localized in the replication factor $\mathrm{C}$ (rfc) 1 gene promoter participate in transcriptional regulation. Subsequent in vitro and in vivo assays in the study showed that DRE sequences of the rfc1 gene are bound to DREF (51). RFC is a five-subunit protein complex involved in DNA replication, of which RFC140 is the largest subunit. The study conducted by Tsuchiya et al (51) also identified via phenotype observation that the $\mathrm{rfcl}$ gene encoding Drosophila RFC140 protein is vital for $G_{1} / S$ cell-cycle progression. Furthermore, through immune-cytochemical experiments, the study found that the cell cycle is closely associated with $\mathrm{rfcl}$ gene expression. These findings indicate that DREF effectively regulates the $\mathrm{rfcl}$ promoter involved in $\mathrm{G}_{1} / \mathrm{S}$ cell-cycle regulation. Additionally, the DRE/DREF system has been found to regulate several Drosophila genes associated with cell cycle progression, including dE2F1, cyclin A and D-myb for progression through the $S$ phase $(32,33,52)$, and D-raf for progression through the $\mathrm{G}_{1}$ and $\mathrm{M}$ phases (35).

It has been reported that DRE/DREF regulates the transcription of the Drosophila osa and moira (mor) genes (53). These two genes encode subunits of the brahma (BRM) complex, which is an ATP-dependent chromatin remodeling complex conserved from yeast to humans $(54,55)$. The BRM complex is thought to negatively regulate entry into the $S$ phase whereas, by contrast, DREF promotes $G_{1} / S$ and $\mathrm{S}$ phase progression. Thus, DREF functions simultaneously as a negative and positive regulator of $\mathrm{G}_{1} / \mathrm{S}$ progression. This type of regulation may inhibit excess induction of the $S$ phase when cell cycle progression is finely tuned.

Ohshima et al (9) used the western blot technique to examine the fluctuation of ZBED1 protein levels during the cell cycle. In this analysis, they used primary cultures from human embryonic lung fibroblasts (HEL cells) that could easily be transformed to a quiescent state when subjected to serum deprivation and then returned to cell cycling after the addition of $10 \%$ fetal calf serum. Progression in the cell cycle was assessed after the cells were released from serum starvation using propidium iodine staining followed by flow cytometric analysis. The results indicated that ZBED1 expression is induced at the $G_{1} / S$ transition stage, and so potentially plays an important role in $\mathrm{G}_{1} / \mathrm{S}$ progression. Therefore, it is hypothesized that DREF/ZBED1 highly regulates $G_{1} / S$ progression as a transcription factor, thereby promoting cell proliferation.

Apoptosis. Although DREF serves a role in the regulation of cell proliferation, it also activates various apoptosis-inducing genes. Cell proliferation is restricted via the Hippo pathway through cell cycle arrest and the induction of apoptosis (56-58). Notably, it has been reported that the DRE/DREF pathway is required for the transcriptional activation of the hpo gene to positively regulate the Hippo pathway (59). Warts, a tumor suppressor gene that encodes a core kinase in the Hippo pathway, is also controlled through the DRE/DREF pathway (60). Using in vivo chromatin immunoprecipitation assays, DREF was demonstrated to selectively bind to the warts gene promoter region containing DREs, and endogenous warts mRNA expression was shown to be reduced in S2 cells following the knockdown of DREF (60). These findings confirm the association between DRE/DREF and the Hippo pathway. Furthermore, in addition to Hippo pathway-associated genes, DREF also associates with Drosophila myeloid leukemia factor in thorax development to positively regulate the basket gene and thereby activate the JNK signaling pathway $(61,62)$, which induces apoptosis and protects the genome.

Studies indicate that the regulation of Drosophila p53 (dmp53) gene expression is highly attributable to the binding of the DRE-containing region of dmp53 to the DREF and that DREF affects apoptosis through the transcriptional activation of dmp53 (63). The dmp53 gene has limited sequence similarity to the human p53 gene (64); however, the function and structure of p53 are conserved in flies and mammals $(65,66)$. P53 is a critical regulatory protein required for diverse cellular metabolic processes, including cell cycle arrest, DNA repair and apoptosis (67). Nearly all common human cancers possess mutations or loss of function of the p53 gene $(68,69)$. The p53 gene, regarded as the 'guardian of the genome', acts as a tumor suppressor gene. Therefore, the finding that DREF positively regulates the expression of the dmp53 gene indicates the role of the former in the fine-tuning of cell proliferation.

Through the database searching of other tumor suppressor genes, including Rbf, APC, Brca2, NF1 and Vhl, DRE and DRE-like sequences have been identified to be regulated by DREF in upstream regions of Drosophila (63). The activation of oncogenes such as Ras or Myc through increased proliferation simultaneously induces apoptosis (70). Apoptosis then represses inappropriate cell proliferation, a function that acts as a mechanism to prevent the proliferation of damaged cells (71). Therefore, DREF is potentially critical in maintaining tissue kinetics and the balance between cell proliferation and apoptosis.

Differentiation. For cells to shift from a proliferating state to a resting state, it is necessary to coordinatively shut down several cell proliferation-associated genes. This process is controlled through differentiation signals. DREF as a major transcription factor of proliferation-related genes is thus a potential mediator of this repression (72). Some differentiation signals have been found to target DRE/DREF. The zerknullt (zen) gene is expressed in the dorsal region of the early embryo at the cellular blastoderm stage. It encodes a homeodomain-containing protein Zen that participates in differentiation of the optic lobe and the amnioserosa (73). Notably, when DREF activity is reduced, Zen expression in cultured cells represses DRE-containing genes (74). Therefore, the DRE/DREF system appears to be a point of intersection between growth and differentiation signaling pathways. Another homeodomain protein, Distal-less (Dll), has been 
found to negatively regulate Drosophila DREF activity (75). The DNA-binding domain of DREF was revealed to selectively bind to Dll and thereby inhibit its transcriptional activity via electrophoretic mobility shift assays. Co-immunoprecipitation assays for DIl and DREF were also conducted; however, they did not yield positive results, indicating that interactions between Dll and DREF may be transient.

DREF has been shown to bind to its DRE sequence thereby promoting the expression of dPCNA in cell proliferation, whereas Cut, which is a Drosophila homolog of the mammalian CCAAT-displacement protein (CDP)/Cux, functions as a transcriptional repressor of the dPCNA gene by binding to the promoter region during cell differentiation (76). The dPCNA promoter was shown to be activated by DREF and repressed by Cut via the measurement of dPCNA promoter activity in transient luciferase expression assays. Moreover, Cut and DREF were demonstrated to be localized in genomic regions containing the dPCNA promoter, which initiates Cut-induced differentiation, using chromatin immunoprecipitation assays. These findings indicate that the DRE sequence binds to DREF during cell proliferation, which causes the state of expression of the dPCNA gene to become 'on'. The dPCNA promoter recruits $C u t$ to its region when the cell is in a differentiated state. Cut then associates with other factors to inhibit expression of dPCNA gene. Therefore, we suggest that during cell proliferation, the differentiation-coupled reduction could be linked to DREF, which is a key regulatory factor for proliferation-associated genes.

\section{ZBED1 as a transcription factor for cell proliferation}

To examine whether ZBED1 regulates human DNA replication-related genes, Ohshima et al (9) assessed the histone H1 gene, the expression of which is stringently coupled with DNA replication. This gene has a single 10-bp sequence in its promoter region that completely matches with the ZBED1 binding sequence $(77,78)$. Co-transfection experiments indicate that the activity of the human histone $\mathrm{H} 1$ gene promoter is stimulated when it specifically binds to ZBED1, and RNA interference experiments targeting ZBED1 indicate that transcription of the histone $\mathrm{H} 1$ gene is likely under the control of ZBED1 in the $\mathrm{G}_{1} / \mathrm{S}$ phase during the cell cycle (9).

Notably, a study established that 22 of the 79 human ribosomal protein (RP) genes have sequences similar to that of hDRE in their transcriptional start sites $<200$ bp upstream. The study also demonstrated that ZBED1 potentially binds to the hDRE-like sequences in the RP genes in vivo and in vitro, and the hDRE-like sequences function as positive elements for RP gene transcription (79). In a similar manner to ZBED1 expression, RP gene expression is enhanced in the late $G_{1}$ to $S$ phases, whereas a reduction in the expression of the RP gene occurs when ZBED1 is depleted, thus impairing cell proliferation and the $G_{1} / S$ transition in normal human fibroblasts. These findings indicate that ZBED1 significantly regulates cell proliferation at the transcriptional level via the histone H1 and several RP genes. Also, ZBED1 has a key role in the cell cycle-dependent regulation of these genes. Furthermore, a study by Yamashita et al (80) explored the underlying transcriptional regulation mechanism and demonstrated that ZBED1 has a slight ubiquitin-like modifier (SUMO) ligase activity and may stimulate transcriptional activation by specifically SUMOylating Mi2 $\alpha$. This results in the dissociation of Mi2 $\alpha$ from the gene loci, thus maintaining active states of housekeeping target genes for ZBED1.

\section{Conclusions and future perspectives}

The present review highlights that Drosophila DREF has different structural features than its human ortholog ZBED1. However, there are notable similarities between the two species based on protein functions associated with, for example, the regulation of DNA replication, chromatin structure, protein synthesis and the cell cycle. Furthermore, ZBED1 potentially participates in the regulation of the expression of housekeeping genes within the mammalian genome. Also, the potential regulatory sites of different genes for DREF binding are likely to be conserved between Drosophila and humans. Therefore, numerous other genes may be regulated similarly in humans via the ZBED1 pathway. However, further research is essential to identify substrates of ZBED1 other than Mi2 $\alpha$ in order to clarify the underlying molecular mechanism and comprehensively analyze the effect of ZBED1 in humans. Additionally, ZBED3 and ZBED6 are associated with tumor development. A recent study has shown that ZBED1 is upregulated in gastric cancer cells, thereby promoting their proliferation and decreasing their chemosensitivity, although the molecular mechanism requires full elucidation (81). Therefore, it is concluded that ZBED1 may be a novel cancer biomarker and therapeutic target in the future. However, further investigation is required to verify this.

\section{Acknowledgements}

Not applicable.

\section{Funding}

This study was supported by the National Natural Science Foundation of China (grant no. 81402458), the Basic Research Project of Shanxi Province (grant no. 2014021037-4), the Graduate Program of Scientific Research Foundation of Yunnan Provincial Department of Education (grant no. 2016YJS057) and the Science and Technology Research Project of Colleges and Universities of Hebei Province (grant no. QN2020234).

\section{Availability of data and materials}

Not applicable.

\section{Authors' contributions}

YJ, RL, XS and GZ conceived and designed the review. YJ, $\mathrm{ZZ}, \mathrm{JR}, \mathrm{XS}$ and GZ were involved in the collection and collation of references. YJ and $\mathrm{ZZ}$ drew the figures. YJ, RL and GZ wrote the manuscript. All authors read and approved the final manuscript.

\section{Ethics approval and consent to participate}

Not applicable. 


\section{Patient consent for publication}

Not applicable.

\section{Competing interests}

The authors declare that they have no competing interests.

\section{References}

1. Lander ES, Linton LM, Birren B, Nusbaum C, Zody MC Baldwin J, Devon K, Dewar K, Doyle M, FitzHugh W, et al: Initial sequencing and analysis of the human genome. Nature 409 860-921, 2001.

2. Razin SV, Borunova VV, Maksimenko OG and Kantidze OL: Cys2His2 zinc finger protein family: Classification, functions, and major members. Biochemistry (Mosc) 77: 217-226, 2012.

3. Markljung E, Jiang L, Jaffe JD, Mikkelsen TS, Wallerman O, Larhammar M, Zhang X, Wang L, Saenz-Vash V, Gnirke A, et al ZBED6, a novel transcription factor derived from a domesticated DNA transposon regulates IGF2 expression and muscle growth. PLoS Biol 7: e1000256, 2009.

4. Akhtar Ali M, Younis S, Wallerman O, Gupta R, Andersson L and Sjöblom T: Transcriptional modulator ZBED6 affects cell cycle and growth of human colorectal cancer cells. Proc Natl Acad Sci USA 112: 7743-7748, 2015.

5. Chen T, Li M, Ding Y, Zhang LS, Xi Y, Pan WJ, Tao DL, Wang JY and Li L: Identification of zinc-finger BED domain-containing 3 (Zbed3) as a novel Axin-interacting protein that activates $\mathrm{Wnt} /$ beta-catenin signaling. J Biol Chem 284: 6683-6689, 2009.

6. Fan C, Jiang G, Zhang X, Miao Y, Lin X, Luan L, Xu Z, Zhang Y, Zhao H, Liu D, et al: Zbed3 contributes to malignant phenotype of lung cancer via regulating $\beta$-catenin and P120-catenin 1. Mol Carcinog 1 (54 Suppl): E138-E147, 2015.

7. Saghizadeh M, Akhmedov NB, Yamashita CK, Gribanova Y, Theendakara V, Mendoza E, Nelson SF, Ljubimov AV and Farber DB: ZBED4, a BED-type zinc-finger protein in the cones of the human retina. Invest Ophthalmol Vis Sci 50: 3580-3588, 2009.

8. Mokhonov VV, Theendakara VP, Gribanova YE, Ahmedli NB and Farber DB: Sequence-specific binding of recombinan Zbed4 to DNA: Insights into Zbed4 participation in gene transcription and its association with other proteins. PLoS One 7: e35317, 2012.

9. Ohshima N, Takahashi M and Hirose F: Identification of a human homologue of the DREF transcription factor with a potential role in regulation of the histone $\mathrm{H} 1$ gene. J Biol Chem 278 : 22928-22938, 2003.

10. Deininger PL and Batzer MA: Mammalian retroelements. Genome Res 12: 1455-1465, 2002.

11. Sinzelle L, Izsvak Z and Ivics Z: Molecular domestication of transposable elements: From detrimental parasites to useful host genes. Cell Mol Life Sci 66: 1073-1093, 2009.

12. van de Lagemaat LN, Landry JR, Mager DL and Medstrand P: Transposable elements in mammals promote regulatory variation and diversification of genes with specialized functions. Trends Genet 19: 530-536, 2003

13. Jordan IK, Rogozin IB, Glazko GV and Koonin EV: Origin of a substantial fraction of human regulatory sequences from transposable elements. Trends Genet 19: 68-72, 2003.

14. Kashkush K, Feldman M and Levy AA: Transcriptional activation of retrotransposons alters the expression of adjacent genes in wheat. Nat Genet 33: 102-106, 2003.

15. Feschotte $\mathrm{C}$ and Pritham EJ: DNA transposons and the evolution of eukaryotic genomes. Annu Rev Genet 41: 331-368, 2007.

16. Volff JN: Turning junk into gold: Domestication of transposable elements and the creation of new genes in eukaryotes. Bioessays 28: 913-922, 2006

17. Arensburger P, Hice RH, Zhou L, Smith RC, Tom AC, Wright JA, Knapp J, O'Brochta DA, Craig NL and Atkinson PW: Phylogenetic and functional characterization of the hAT transposon superfamily. Genetics 188: 45-57, 2011.

18. Hayward A, Ghazal A, Andersson G, Andersson L and Jern P: ZBED evolution: Repeated utilization of DNA transposons as regulators of diverse host functions. PLoS One 8: e59940, 2013 .
19. Hirose F, Yamaguchi M, Nishida Y, Masutani M, Miyazawa H, Hanaoka $\mathrm{F}$ and Matsukage A: Structure and expression during development of Drosophila melanogaster gene for DNA polymerase alpha. Nucleic Acids Res 19: 4991-4998, 1991.

20. Hirose F, Yamaguchi M, Handa H, Inomata $Y$ and Matsukage $A$ : Novel 8-base pair sequence (Drosophila DNA replication-related element) and specific binding factor involved in the expression of Drosophila genes for DNA polymerase alpha and proliferating cell nuclear antigen. J Biol Chem 268: 2092-2099, 1993.

21. Hirose F, Yamaguchi M, Kuroda K, Omori A, Hachiya T, Ikeda M, Nishimoto $Y$ and Matsukage A: Isolation and characterization of cDNA for DREF, a promoter-activating factor for Drosophila DNA replication-related genes. J Biol Chem 271: 3930-3937, 1996

22. Takahashi Y, Hirose F, Matsukage A and Yamaguchi M: Identification of three conserved regions in the DREF transcription factors from Drosophila melanogaster and Drosophila virilis. Nucleic Acids Res 27: 510-516, 1999.

23. Smit AF: Interspersed repeats and other mementos of transposable elements in mammalian genomes. Curr Opin Genet Dev 9: 657-663, 1999.

24. Aravind L: The BED finger, a novel DNA-binding domain in chromatin-boundary-element-binding proteins and transposases. Trends Biochem Sci 25: 421-423, 2000.

25. HartCM,Cuvier O and Laemmli UK: Evidence for an antagonistic relationship between the boundary element-associated factor BEAF and the transcription factor DREF. Chromosoma 108: 375-383, 1999.

26. Yamashita D, Komori H, Higuchi Y, Yamaguchi T, Osumi T and Hirose F: Human DNA replication-related element binding factor (hDREF) self-association via hATC domain is necessary for its nuclear accumulation and DNA binding. J Biol Chem 282: 7563-7575, 2007

27. Essers L, Adolphs RH and Kunze R: A highly conserved domain of the maize activator transposase is involved in dimerization. Plant Cell 12: 211-224, 2000.

28. Michel K, O'Brochta DA and Atkinson PW: The C-terminus of the Hermes transposase contains a protein multimerization domain. Insect Biochem Mol Biol 33: 959-970, 2003.

29. Matsukage A, Hirose F, Yoo MA and Yamaguchi M: The DRE/DREF transcriptional regulatory system: A master key for cell proliferation. Biochim Biophys Acta 1779: 81-89, 2008.

30. Tue NT, Yoshioka Y, Mizoguchi M, Yoshida H, Zurita M and Yamaguchi M: DREF plays multiple roles during Drosophila development. Biochim Biophys Acta Gene Regul Mech 1860: 705-712, 2017.

31. Matsukage A, Hirose F, Hayashi Y, Hamada K and Yamaguchi M: The DRE sequence TATCGATA, a putative promoter-activating element for Drosophila melanogaster cell-proliferation-related genes. Gene 166: 233-236, 1995.

32. Sawado T, Hirose F, Takahashi Y, Sasaki T, Shinomiya T, Sakaguchi K, Matsukage A and Yamaguchi M: The DNA replication-related element (DRE)/DRE-binding factor system is a transcriptional regulator of the Drosophila E2F gene. J Biol Chem 273: 26042-26051, 1998

33. Ohno K, Hirose F, Sakaguchi K, Nishida Y and Matsukage A Transcriptional regulation of the Drosophila CycA gene by the DNA replication-related element (DRE) and DRE binding factor (DREF). Nucleic Acids Res 24: 3942-3946, 1996.

34. Takahashi Y, Yamaguchi M, Hirose F, Cotterill S, Kobayashi J, Miyajima $S$ and Matsukage A: DNA replication-related elements cooperate to enhance promoter activity of the drosophila DNA polymerase alpha 73-kDa subunit gene. J Biol Chem 271: 14541-14547, 1996.

35. Ryu JR, Choi TY, Kwon EJ, Lee WH, Nishida Y, Hayashi Y, Matsukage A, Yamaguchi $M$ and Yoo MA: Transcriptional regulation of the Drosophila-raf proto-oncogene by the DNA replication-related element (DRE)/DRE-binding factor (DREF) system. Nucleic Acids Res 25: 794-799, 1997.

36. Zabidi MA, Arnold CD, Schernhuber K, Pagani M, Rath M, Frank $\mathrm{O}$ and Stark A: Enhancer-core-promoter specificity separates developmental and housekeeping gene regulation. Nature 518: 556-559, 2015.

37. Bird AP: CpG-rich islands and the function of DNA methylation. Nature 321: 209-213, 1986.

38. Argentaro A, Yang JC, Chapman L, Kowalczyk MS, Gibbons RJ, Higgs DR, Neuhaus D and Rhodes D: Structural consequences of disease-causing mutations in the ATRX-DNMT3-DNMT3L (ADD) domain of the chromatin-associated protein ATRX. Proc Natl Acad Sci USA 104: 11939-11944, 2007. 
39. Valadez-Graham V, Yoshioka Y, Velazquez O, Kawamori A, Vazquez M, Neumann A, Yamaguchi $M$ and Zurita $M$ : $\mathrm{XNP} / \mathrm{dATRX}$ interacts with DREF in the chromatin to regulate gene expression. Nucleic Acids Res 40: 1460-1474, 2012.

40. Hart CM, Zhao K and Laemmli UK: The scs' boundary element: Characterization of boundary element-associated factors. Mol Cell Biol 17: 999-1009, 1997.

41. Royzman I, Whittaker AJ and Orr-Weaver TL: Mutations in Drosophila DP and E2F distinguish G1-S progression from an associated transcriptional program. Genes Dev 11: 1999-2011, 1997.

42. Brehm A, Langst G, Kehle J, Clapier CR, Imhof A, Eberharter A, Muller J and Becker PB: dMi-2 and ISWI chromatin remodelling factors have distinct nucleosome binding and mobilization properties. EMBO J 19: 4332-4341, 2000.

43. Hirose F, Ohshima N, Kwon EJ, Yoshida $\mathrm{H}$ and Yamaguchi $\mathrm{M}$ : Drosophila Mi-2 negatively regulates dDREF by inhibiting its DNA-binding activity. Mol Cell Biol 22: 5182-5193, 2002.

44. Ida H, Yoshida H, Nakamura K and Yamaguchi M: Identification of the Drosophila eIF4A gene as a target of the DREF transcription factor. Exp Cell Res 313: 4208-4220, 2007.

45. Linder P, Lasko PF, Ashburner M, Leroy P, Nielsen PJ, Nishi K, Schnier J and Slonimski PP: Birth of the D-E-A-D box. Nature 337: 121-122, 1989.

46. Blum S, Schmid SR, Pause A, Buser P, Linder P, Sonenberg N and Trachsel H: ATP hydrolysis by initiation factor 4A is required for translation initiation in Saccharomyces cerevisiae. Proc Natl Acad Sci USA 89: 7664-7668, 1992.

47. Pestova TV, Kolupaeva VG, Lomakin IB, Pilipenko EV Shatsky IN, Agol VI and Hellen CU: Molecular mechanisms of translation initiation in eukaryotes. Proc Natl Acad Sci USA 98: 7029-7036, 2001.

48. Pestova TV, Shatsky IN and Hellen CU: Functional dissection of eukaryotic initiation factor 4F: The 4A subunit and the central domain of the $4 \mathrm{G}$ subunit are sufficient to mediate internal entry of 43S preinitiation complexes. Mol Cell Biol 16: 6870-6878, 1996.

49. Phuong Thao DT, Ida H, Yoshida $H$ and Yamaguchi $M$ : Identification of the Drosophila skpA gene as a novel target of the transcription factor DREF. Exp Cell Res 312: 3641-3650, 2006.

50. Seol JH, Shevchenko A, Shevchenko A and Deshaies RJ: Skpl forms multiple protein complexes, including RAVE, a regulator of V-ATPase assembly. Nat Cell Biol 3: 384-391, 2001.

51. Tsuchiya A, Inoue YH, Ida H, Kawase Y, Okudaira K, Ohno K, Yoshida $\mathrm{H}$ and Yamaguchi M: Transcriptional regulation of the Drosophila rfc1 gene by the DRE-DREF pathway. FEBS J 274 1818-1832, 2007.

52. Sharkov NV, Ramsay G and Katzen AL: The DNA replication-related element-binding factor (DREF) is a transcriptional regulator of the Drosophila myb gene. Gene 297: 209-219, 2002.

53. Nakamura $\mathrm{K}$, Ida $\mathrm{H}$ and Yamaguchi $\mathrm{M}$ : Transcriptional regulation of the Drosophila moira and osa genes by the DREF pathway. Nucleic Acids Res 36: 3905-3915, 2008.

54. Mohrmann L and Verrijzer CP: Composition and functional specificity of SWI2/SNF2 class chromatin remodeling complexes. Biochim Biophys Acta 1681: 59-73, 2005.

55. Bouazoune $\mathrm{K}$ and Brehm A: ATP-dependent chromatin remodeling complexes in Drosophila. Chromosome Res 14: 433-449, 2006.

56. He M, Zhou Z, Shah AA, Hong Y, Chen Q and Wan Y: New insights into posttranslational modifications of Hippo pathway in carcinogenesis and therapeutics. Cell Div 11: 4, 2016.

57. Meng Z, Moroishi T and Guan KL: Mechanisms of Hippo pathway regulation. Genes Dev 30: 1-17, 2016.

58. Ye S and Eisinger-Mathason TS: Targeting the Hippo pathway: Clinical implications and therapeutics. Pharmacol Res 103: 270-278, 2016.

59. Vo N, Horii T, Yanai H, Yoshida H and Yamaguchi M: The Hippo pathway as a target of the Drosophila DRE/DREF transcriptional regulatory pathway. Sci Rep 4: 7196, 2014.

60. Fujiwara S, Ida H, Yoshioka Y, Yoshida $H$ and Yamaguchi M: The warts gene as a novel target of the Drosophila DRE/DREF transcription pathway. Am J Cancer Res 2: 36-44, 2012.

61. Yanai H, Yoshioka Y, Yoshida H, Nakao Y, Plessis A and Yamaguchi M: Drosophila myeloid leukemia factor acts with DREF to activate the JNK signaling pathway. Oncogenesis 3: e98, 2014.
62. Yoshioka Y, Nguyen TT, Fujiwara S, Matsuda R, Valadez-Graham V, Zurita M and Yamaguchi M: Drosophila DREF acting via the JNK pathway is required for thorax development. Genesis 50: 599-611, 2012.

63. Trong-Tue N, Thao DT and Yamaguchi M: Role of DREF in transcriptional regulation of the Drosophila p53 gene. Oncogene 29: 2060-2069, 2010.

64. Brodsky MH, Nordstrom W, Tsang G, Kwan E, Rubin GM and Abrams JM: Drosophila p53 binds a damage response element at the reaper locus. Cell 101: 103-113, 2000.

65. Jin S, Martinek S, Joo WS, Wortman JR, Mirkovic N, Sali A, Yandell MD, Pavletich NP, Young MW and Levine AJ: Identification and characterization of a p53 homologue in Drosophila melanogaster. Proc Natl Acad Sci USA 97: 7301-7306, 2000.

66. Ollmann M, Young LM, Di Como CJ, Karim F, Belvin M, Robertson S, Whittaker K, Demsky M, Fisher WW, Buchman A, et al: Drosophila p53 is a structural and functional homolog of the tumor suppressor p53. Cell 101: 91-101, 2000.

67. Nicolai S, Rossi A, Di Daniele N, Melino G, Annicchiarico-Petruzzelli $M$ and Raschella G: DNA repair and aging: The impact of the p53 family. Aging (Albany NY) 7: 1050-1065, 2015

68. Kim S and An SS: Role of p53 isoforms and aggregations in cancer. Medicine (Baltimore) 95: e3993, 2016.

69. Chen J: The Cell-cycle arrest and apoptotic functions of p53 in tumor initiation and progression. Cold Spring Harb Perspect Med 6: a026104, 2016.

70. Green DR and Evan GI: A matter of life and death. Cancer Cell 1: 19-30, 2002.

71. Wu S, Huang J, Dong J and Pan D: hippo encodes a Ste-20 family protein kinase that restricts cell proliferation and promotes apoptosis in conjunction with salvador and warts. Cell 114: 445-456, 2003.

72. Matsukage A, Hirose F and Yamaguchi M: Transcriptional regulation of DNA replication-related genes in cell growth, differentiation and oncogenesis. Jpn J Cancer Res 85: 1-8, 1994.

73. Doyle HJ, Kraut R and Levine M: Spatial regulation of zerknullt: A dorsal-ventral patterning gene in Drosophila. Genes Dev 3: 1518-1533, 1989.

74. Hirose F, Yamaguchi M and Matsukage A: Repression of regulatory factor for Drosophila DNA replication-related gene promoters by zerknullt homeodomain protein. J Biol Chem 269: 2937-2942, 1994

75. Hayashi Y, Kato M, Seto $\mathrm{H}$ and Yamaguchi M: Drosophila distal-less negatively regulates $\mathrm{dDREF}$ by inhibiting its DNA binding activity. Biochim Biophys Acta 1759: 359-366, 2006.

76. Seto H, Hayashi Y, Kwon E, Taguchi O and Yamaguchi M: Antagonistic regulation of the Drosophila PCNA gene promoter by DREF and Cut. Genes Cells 11: 499-512, 2006

77. van Wijnen AJ, Wright KL, Massung RF, Gerretsen M, Stein JL and Stein GS: Two target sites for protein binding in the promoter region of a cell cycle regulated human $\mathrm{H} 1$ histone gene. Nucleic Acids Res 16: 571-592, 1988

78. Albig W, Meergans T and Doenecke D: Characterization of the $\mathrm{H} 1.5$ gene completes the set of human $\mathrm{H} 1$ subtype genes. Gene 184: 141-148, 1997.

79. Yamashita D, Sano Y, Adachi Y, Okamoto Y, Osada H, Takahashi T, Yamaguchi T, Osumi T and Hirose F: hDREF regulates cell proliferation and expression of ribosomal protein genes. Mol Cell Biol 27: 2003-2013, 2007.

80. Yamashita D, Moriuchi T, Osumi T and Hirose F: Transcription Factor hDREF Is a Novel SUMO E3 Ligase of Mi2 $\alpha$. J Biol Chem 291: 11619-11634, 2016.

81. Jiang S, Wang Y, Xiong Y, Feng Y, Tang J and Song R: High expression of ZBED1 affects proliferation and apoptosis in gastric cancer. Int J Clin Exp Pathol 11: 4019-4025, 2018.

This work is licensed under a Creative Commons Attribution-NonCommercial-NoDerivatives 4.0 International (CC BY-NC-ND 4.0) License. 